\title{
Differentiation between gastrointestinal schwannomas and gastrointestinal stromal tumors by computed tomography
}

\author{
MING-YAN HE ${ }^{1 *}$, RONG ZHANG ${ }^{2 *}$, ZHENPENG PENG ${ }^{1 *}$, YIN LI $^{3}$, \\ LING XU $^{4}$, MENGJIE JIANG ${ }^{1}$, ZI-PING LI ${ }^{1}$ and SHI-TING FENG ${ }^{1}$

\begin{abstract}
${ }^{1}$ Department of Radiology, The First Affiliated Hospital; ${ }^{2}$ Department of Radiology, The Tumor Hospital; ${ }^{3}$ Department of ${ }^{4}$ Faculty of Medicine and Dentistry, University of Western Australia, Perth, WA 6009, Australia
\end{abstract} \\ Gastrointestinal Surgery, The First Affiliated Hospital, Sun Yat-Sen University, Guangzhou, Guangdong 510080, P.R. China;
}

Received March 9, 2016; Accepted January 31, 2017

DOI: $10.3892 / \mathrm{ol} .2017 .5955$

\begin{abstract}
The aim of the present study was to identify computed tomography (CT) features to assist in differentiating gastrointestinal schwannomas from gastrointestinal stromal tumors (GISTs). CT images of gastrointestinal schwannomas $(n=15)$ and GISTs $(n=50)$ were analyzed. The absolute CT values of tumor/aorta during plain scan/arterial phase/venous phase were recorded as tumor plain scan (Tp)/aorta plain scan (Ap), tumor arterial phase (Ta)/aorta arterial phase (Aa) and tumor venous phase (Tv)/aorta venous phase (Av), respectively, and normalized $\mathrm{CT}$ values of the three phases were calculated as $\mathrm{Sp}=\mathrm{Tp} / \mathrm{Ap}, \mathrm{Sa}=\mathrm{Ta} / \mathrm{Aa}$ and $\mathrm{Sv}=\mathrm{Tv} / \mathrm{Av}$, respectively. The difference in tumor CT value between arterial and venous phases was calculated and recorded as Tv-a. CT data including tumor size, contour, margin, growth pattern, presence of calcification, cystic change, hemorrhage, ulceration, perilesional lymph nodes (PLNs), local invasion to surrounding structures, metastasis, ascites, vasculatures, enhancement pattern/degree,
\end{abstract}

Correspondence to: Dr Zi-Ping Li or Dr Shi-Ting Feng, Department of Radiology, The First Affiliated Hospital, Sun Yat-Sen University, 58 Second Zhongshan Road, Yuexiu, Guangzhou, Guandong 510080, P.R. China

E-mail: liziping163@163.com

E-mail: fst1977@163.com

*Contributed equally

Abbreviations: CT, computed tomography; GIST, gastrointestinal stromal tumors; Tp, tumor plain scan; Ap, aorta plain scan; Ta, tumor arterial phase; Aa, aorta arterial phase; Tv, tumor venous phase; Av, aorta venous phase; Sp, tumor plain scan over aorta plain scan; Sa, tumor arterial phase over aorta arterial phase; Sv, tumor venous phase over aorta venous phase; ROC, receiver operating characteristic; Tv-a, difference between tumor venous and arterial phases; PLN, perilesional lymph nodes; AUC, area under curve; ROI, region of interest; HU, Hounsfield units

Key words: gastrointestinal schwannoma, gastrointestinal stromal tumor, computed tomography, differential diagnosis
$\mathrm{Tp} / \mathrm{Ta} / \mathrm{Tv}$ and $\mathrm{Sp} / \mathrm{Sa} / \mathrm{Sv}$ were evaluated for each patient. Receiver operating characteristic (ROC) curve analysis was used to assess the ability of the CT data to differentiate gastrointestinal schwannomas from GISTs. Compared with GISTs, gastrointestinal schwannomas more frequently demonstrated round contouring, relatively smaller tumor size, a homogeneous enhancement pattern, with the presence of PLNs and a higher level of vasculature $(\mathrm{P}<0.05)$, whilst the presence of cystic changes were more common in GISTs compared with gastrointestinal schwannomas $(\mathrm{P}<0.05)$. The Sa, Ta and Tv-a of gastrointestinal schwannomas were less compared with those of GISTs $(\mathrm{P}<0.05)$. The difference in margin, growth pattern, intra-tumoral calcifications and hemorrhage were insignificant $(\mathrm{P}>0.05)$. ROC analysis indicated that tumor size, cystic change, the presence of PLNs, tumor enhancement pattern and $\mathrm{Sa}$ demonstrated improved diagnostic potential compared with others [area under the curve (AUC) >0.7], amongst which cystic change demonstrated the best diagnostic ability (AUC=0.82). Size exhibited the highest sensitivity, $90 \%$, and cystic change, Sa exhibited the best specificity, $87 \%$. Quantitative analysis indicated that certain features aided the differentiation between gastrointestinal schwannomas and GISTs using CT imaging.

\section{Introduction}

Schwannoma are benign tumors arising from Schwann cells in the sheaths of peripheral nerves. Schwannoma are homogeneous tumors and may occur in any tissue of the body. The head and neck region is the most prevalent location for schwannoma to occur; they are rarely observed in the gastrointestinal tract (1). Conventional schwannoma usually arise from peripheral skin nerves and connective tissue, whereas gastrointestinal schwannoma tumors are derived from Schwann cells of the Auerbach's plexus within the gastrointestinal tract wall (1-3) and were first reported by Daimaru et al in 1988 (1). As benign mesenchymal tumors, schwannomas only account for 1-2\% of alimentary tract mesenchymal tumors $(2,3)$. By contrast, gastrointestinal stromal tumors (GISTs), another type of mesenchymal tumor, are the most common type of submucosal tumor in the alimentary tract. GISTs and gastrointestinal schwannomas are typically observed in $40-60$ year old patients $(4,5)$ 
and demonstrate similar computed tomography (CT) imaging characteristics and clinical symptoms $(6,7)$. The biological behaviors, appropriate treatments and prognoses vary between GIST and gastrointestinal schwannomas. Gastrointestinal schwannomas generally grow slowly and are associated with an excellent prognosis compared with GIST (8). The benefit of surgical resection for benign gastrointestinal tumors remains debatable (8-10). By contrast, GISTs are potentially malignant, and early surgical resection is recommended, regardless of the tumor size. Furthermore, patients with high-risk GISTs should receive imatinib treatment subsequent to surgical resection (11). Therefore, distinguishing between gastrointestinal schwannomas and GISTs is important to determine whether surgical resection is required as part of treatment.

Unlike mucosal gastrointestinal tumors, including gastric carcinoma and colorectal carcinoma, gastrointestinal mesenchymal tumors are difficult to definitively diagnose with endoscopy prior to surgery (9). In patients with gastrointestinal mesenchymal tumors, endoscopy typically reveals undamaged mucosa and an insert image suggesting extrinsic compression of the gastrointestinal lumen. However, all mesenchymal tumors have similar endoscopic image characteristics (9). In addition, a biopsy guided by endoscopy may be insufficient to collect the amount of tissue required to inform a correct diagnosis (10-12). Thus, endoscopy is insufficient for the specific diagnosis of gastrointestinal mesenchymal tumors.

As modern CT imaging is associated with a rapid scan speed, improved resolution improvement and compatibility with contrast media, it has been demonstrated as an important and valuable tool for preoperative diagnosis, and the staging of gastrointestinal tract tumors (12). However, differentiating between GISTs and gastrointestinal schwannomas prior to surgery remains challenging due to their similar CT appearances, and schwannomas are commonly misdiagnosed as GISTs $(6,12)$. To address this issue, previous investigations have identified that certain CT features, including the density, enhancement pattern, growth pattern and the presence of PLNs, as well as doubling times, may assist in differentiating gastric schwannoma from GIST $(13,14)$. However, the majority of previous studies have focused on certain qualitative CT features that are highly dependent on the experience of the attending radiologists $(13,14)$. Additionally, numerous previous studies had small gastrointestinal schwannoma sample sizes. Therefore, in the present study, qualitative and quantitative analyses were used to identify CT features that may aid the diagnostic differentiation between gastric schwannomas and GIST.

\section{Materials and methods}

Patients. The present study was conducted in accordance with the ethics guidelines for human research, and was compliant with the Health Insurance Portability and Accountability Act (China). The present study received Institutional Review Board and ethics committee approval of the First Affiliated Hospital of Sun Yat-Sen University (Guangzhou, China), and written informed consent was obtained from all patients. Complete clinical, imaging and follow-up data of 50 patients with GIST and 15 patients with gastrointestinal schwannomas who presented between January 2000 and July 2014 and underwent whole abdomen CT and surgical resection were included in the present study. Amongst the patients with gastrointestinal schwannoma, there were 9 females; the mean age was 55.3 years; the age range was 35-74 years, 13 tumors originated in the stomach, 1 in the duodenum and 1 in the transverse colon. Prior to surgery, 12 gastrointestinal schwannomas were misdiagnosed as GISTs, 2 as gastric cancer and 1 as a metastatic tumor. Amongst the 50 patients with GIST, there were 17 females; the mean age was 56.8 years; the age range was 35-39 years, 36 tumors originated in the stomach, 8 in the duodenum, 5 in the jejunum and 1 in the ileum. Histopathological confirmation was obtained from surgical excision in all 65 patients, and all PLNs identified during surgery were fully excised.

CT protocol. Patients fasted for a minimum of $12 \mathrm{~h}$ prior to CT examination. On the day of examination, each patient was provided with $1,600-2,000 \mathrm{ml}$ of $2.5 \%$ oral mannite, administered at $400-500 \mathrm{ml}$ each time with an interval of $15 \mathrm{~min}$, and $500 \mathrm{ml} 2.5 \%$ mannite enema. The CT examination was performed immediately, subsequent to the last oral administration, using the Aquilion 64 CT scanner or the Xpress/SX Multi-detector scanner (Toshiba Medical Systems Corporation, Tokyo, Japan). The patient was positioned supine and scanned from the diaphragm to the level of tuberosity of ischium. For a contrast-enhanced CT, $1.5 \mathrm{ml} / \mathrm{kg}$ iopromide (Ultravist 300 ; Schering, Berlin, Germany) was administrated at a rate of 3-4 ml/sec. The CT scan was obtained 34-37 sec prior to the injection of the contrast agent and 60-70 sec subsequent to the injection of the contrast agent. The scanning parameters were as follows: A beam collimation of $0.5 \mathrm{~mm} \times 64$; tube voltage of $120 \mathrm{kV}$; 200-250 mAs; slice thickness/interval $5 \mathrm{~mm} / 5 \mathrm{~mm}$.

Image analysis. The CT images were evaluated according to the consensus of two radiologists with $>5$ years of experience. Differences in opinion between the two radiologists during initial interpretation were subjected to additional discussion to determine a final conclusion. The masses were evaluated for size, contour (round, quasi-circular or lobulated), growth pattern (endoluminal, exophytic or mixed), margin (well-defined or ill-defined), calcification, cystic change, hemorrhage, ulceration, the presence of PLNs, invasion to other solid organs, metastasis, ascites, tumor vessels, reoccurrence status and enhancement pattern (homogeneous or heterogeneous).

Qualitative analysis in venous phase. If the parameter of the intracavitary tumor extended beyond the margin of the gastrointestinal structures profile, the growth pattern was defined as exophytic. A tumor located within the margin of the gastrointestinal structures was defined as exhibiting an endoluminal growth pattern. If the parameter was across the margins of gastrointestinal structures, it was defined as a mixed growth pattern. A tumor with smooth-edged margins was considered to be well-defined whereas a rough-edged tumor was considered to be ill-defined. High-density regions were defined as 80-200 Hounsfield units (HU) during unenhanced CT scans. Cystic change was defined as instances 
Table I. Clinical symptoms of gastric schwannomas and gastrointestinal stromal tumors.

\begin{tabular}{lcc}
\hline Symptom & Schwannomas & $\begin{array}{c}\text { Gastrointestinal } \\
\text { stromal tumors }\end{array}$ \\
\hline Bellyache & 4 & 7 \\
Abdominal fullness & 5 & 9 \\
or discomfort & & \\
Melanemesis & 2 & 10 \\
Examination data & 1 & 3 \\
Others & 3 & 21 \\
\hline
\end{tabular}



Figure 1. Exophytic growth pattern of a gastrointestinal stromal tumor. Computed tomography scan demonstrating an exophytic lobulated heterogeneous mass with a patchy, low-density region in the gastric antrum.

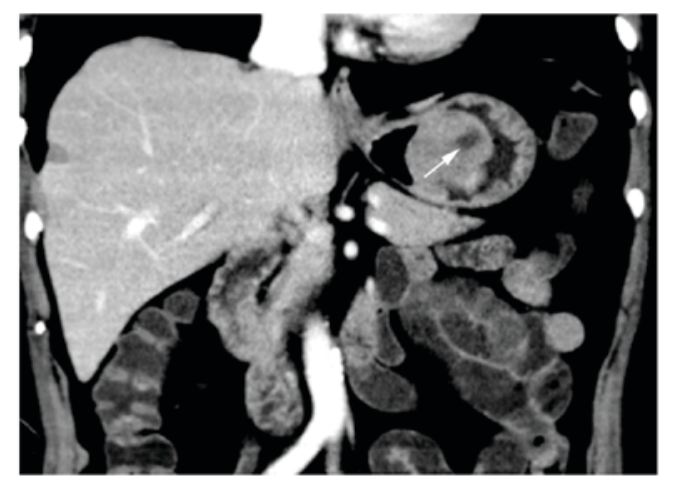

Figure 2. Endoluminal growth pattern of a gastrointestinal stromal tumor. Computed tomography scan demonstrating an endoluminal lobulated heterogeneous mass with a low-density necrosis region under the mucosa of the gastric bottom (arrow)

where there were quasi-circular, watery density regions exhibited in the unenhanced CT scan, and no enhancement pattern detected during the enhanced CT scan. Hemorrhage was defined as a patchy, high-density region detected in the unenhanced CT scan, with CT values between 60 and $80 \mathrm{HU}$ (10). Ulceration was defined as silt- or semi-elliptical-shaped lesions of gastrointestinal mucosa extending to the tumor,

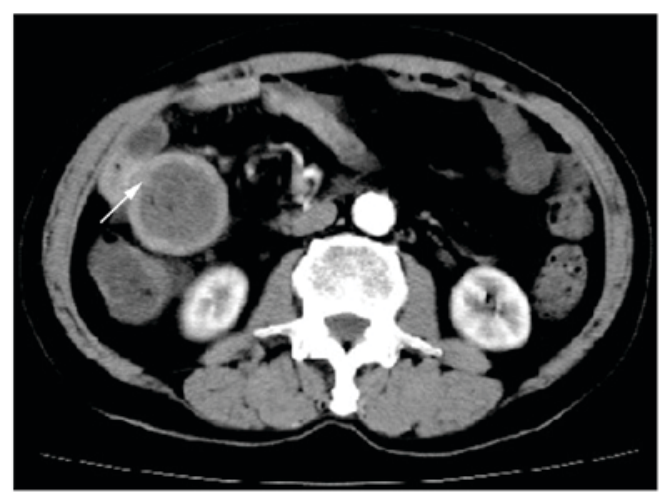

Figure 3. Mixed growth pattern of a gastrointestinal stromal tumor. Transverse computed tomography scan demonstrating a well-defined round mass with moderate enhancement (arrow) in the lesser gastric curvature. The mass revealed an endoluminal and exophytic growth pattern.

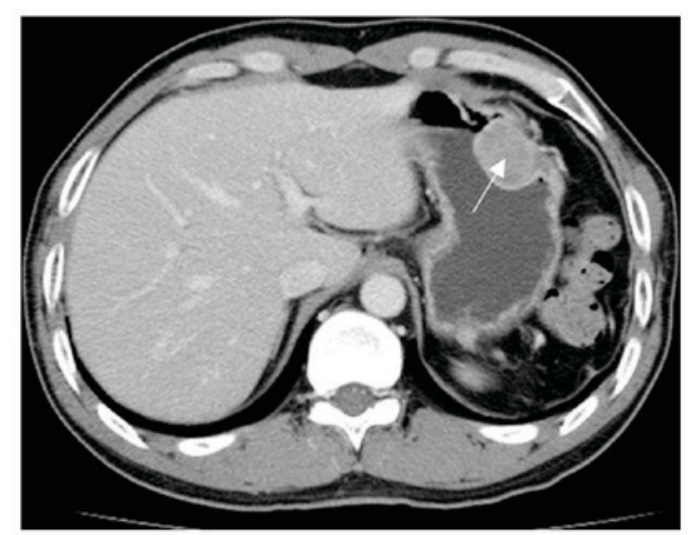

Figure 4. Endoluminal growth pattern of a gastrointestinal schwannoma. Transverse computed tomography scan demonstrating an endoluminal, well-defined, round mass with homogeneous enhancement (arrow).

in which gas or intestinal contrast agent was identified (11). The degree of contrast enhancement was calculated as the difference between venous phase and plain $\mathrm{CT}$ values. If the difference was $<10 \mathrm{HU}$, the tumor was considered to exhibit a mild enhancement pattern; $10-40 \mathrm{HU}$ was considered to be a moderate enhancement pattern and $>40 \mathrm{HU}$ to be a strong enhancement pattern (12). Positive PLNs were defined as lymph nodes surrounding the tumor with a minor axis $>1 \mathrm{~cm}$, or with clear enhancement (6). The size of tumor was determined by measuring the maximal diameter recorded in cross-sectional images, $\geq 5 \mathrm{~cm}$ or $<5 \mathrm{~cm}$.

Quantitative analysis. The center cross-section CT images of the tumor during plain $\mathrm{CT}(\mathrm{Tp})$, arterial phase (Ta) and venous phase (Tv) were selected, and the mean values were recorded using a region of interest (ROI) of those with a diameter from 5 to $15 \mathrm{~mm}$. Blood vessels detectable by the naked eye, necrosis, cystic change, calcification and artifacts were not included in the ROI drawing. The absolute $\mathrm{CT}$ values of the tumor (and aorta) in the same slice during plain scan, arterial phase and venous phase were recorded as Tp (or aorta plain scan, Ap), Ta (aorta arterial phase scan, Aa) and Tv (aorta venous phase scan, Av), respectively. To avoid inaccuracy caused by different injection rates, different injected doses and individual 


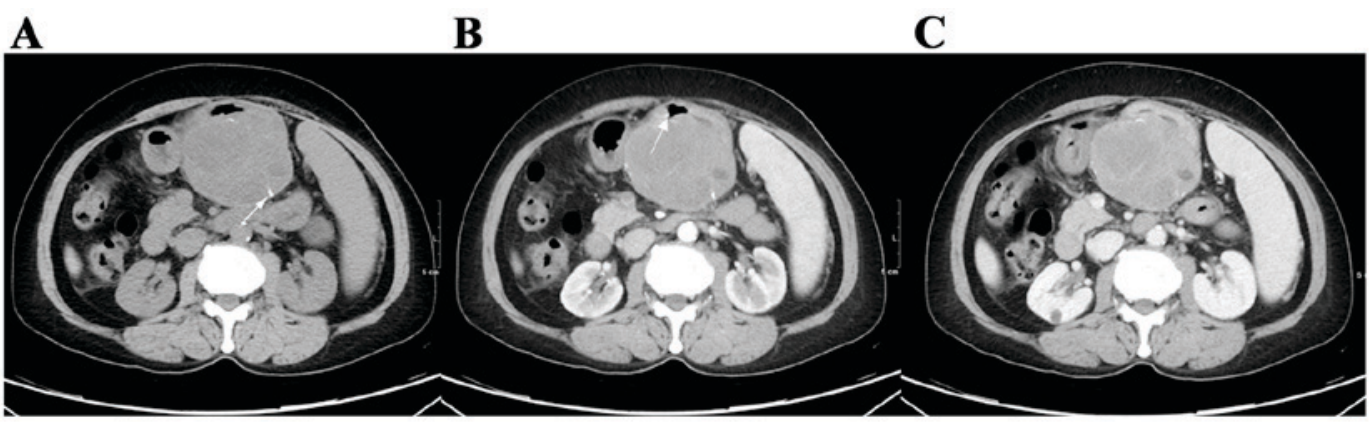

Figure 5. Exophytic growth pattern of a gastrointestinal schwannoma. Computed tomography scan demonstrating an exophytic heterogeneous mass with (A) patchy calcification (arrow), (B) incomplete mucosa, light ulceration (arrow) and (C) necrosis.

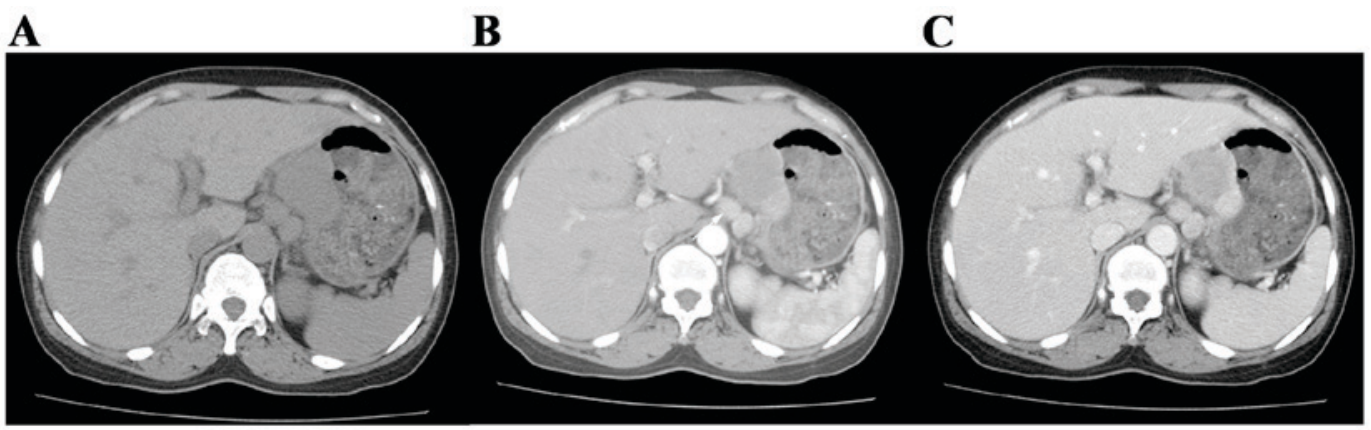

Figure 6. Gastrointestinal schwannoma with perilesional lymph nodes enlargement. Computed tomography scan demonstrating a mass with (A) complete mucosa and (B and C) homogeneously light-moderate enhancement with a lesser gastric curvature. (B and C) Swollen, large lymph nodes were frequently exhibited surrounding the focus, with obvious enhancement (arrow).

circulation levels in different patients, the CT values of tumor, $\mathrm{Tp}$, Ta and Tv, were divided by the corresponding CT values of the abdominal aorta in the same layer, Ap, Aa and Av, to produce normalized CT values, $\mathrm{Sp}$ (Tp/Ap), Sa (Ta/Aa) and $\mathrm{Sv}$ (Tv/Av), respectively. Additionally, the difference in CT value of the tumor between the arterial and venous phase was calculated and recorded as Tv-a.

Statistical analysis. All statistical analyses were performed using SPSS 16.0 (SPSS, Inc., Chicago, IL, USA). Quantitative data was presented as the mean \pm standard deviation and enumeration data was recorded as percentages. An unpaired or Satterthwaite's approximate t-test was performed to compare quantitative data between the groups. A $\chi^{2}$ test or Fisher's exact test was performed to compare enumeration data. $\mathrm{P}<0.05$ was considered to indicate a statistically significant difference.

Receiver operating characteristic (ROC) curve analysis, using a maximum likelihood method, was used to assess the performance of the CT scans in differentiating between gastrointestinal schwannomas and GISTs. The performance was evaluated by calculating the area under the curve (AUC) of the ROC curve. The judgment criteria were as follows: AUC value of 0.5-0.7, low diagnostic value; AUC 0.7-0.9, medium; AUC $>0.9$, high. All CT features with an AUC $>0.7$ were evaluated for specificity and sensitivity. Sensitivity was defined as the true positive rate, which reflects the ability to correctly diagnose patients with the disease. Specificity was defined as the true negative rate, which reflects the ability to correctly identify patients without the disease.

\section{Results}

Clinical characteristics of patients. Patients with gastrointestinal schwannomas or GISTs commonly present with similar clinical symptoms (Table I). CT images of all 15 patients with gastrointestinal schwannomas did not demonstrate the presence of ascites, local invasion to surrounding structures or distant metastasis. PLN in these patients were subsequently assessed, confirming there was no histopathological evidence of metastasis. No recurrence or metastatic lesions were detected at a mean follow-up period of 12.6 months subsequent to surgery. In comparison, the CT images obtained from 4 GISTs patients demonstrated local invasion to the surrounding structures. Distant metastasis was evident in 6 patients, 5 with liver metastasis and 1 with lymphatic metastasis. Recurrence occurred in 2 patients at 14 and 33 months follow-up, respectively.

Comparison of CT data. The differences between gastrointestinal schwannomas and GISTs in tumor margin (Figs. 1-5), growth pattern (Figs. 1-5), intratumoral calcification (Fig. 5) and hemorrhage were not statistically significant. The differences in size $(\mathrm{P}=0.007)$, contour $(\mathrm{P}=0.041)$, cystic change (Fig. 2; $\mathrm{P}<0.0001)$, PLNs (Fig. 6; $\mathrm{P}=0.0007$ ), tumor vessels $(\mathrm{P}=0.0098)$, pattern $(\mathrm{P}=0.0002)$ and degree $(\mathrm{P}=0.005)$ of contrast enhancement were statistically significant (Table II). The Sa $(\mathrm{P}=0.0002)$, Ta $(\mathrm{P}=0.0001)$ and Tv-a $(\mathrm{P}=0.0108)$ of GISTs were significantly higher than gastrointestinal schwannomas in all cases (Figs. 7 and 8). No statistically significant differences were observed in the remaining CT features. 
Table II. CT results of gastric schwannomas and GISTs.

\begin{tabular}{|c|c|c|c|c|c|}
\hline \multirow[b]{2}{*}{$\mathrm{CT}$ results } & \multicolumn{2}{|c|}{ Schwannomas $(n=15)$} & \multicolumn{2}{|c|}{ GISTs $(n=50)$} & \multirow[b]{2}{*}{ P-value } \\
\hline & $\%$ & $\mathrm{n}$ & $\%$ & $\mathrm{n}$ & \\
\hline Size & & & & & $0.007^{\mathrm{a}}$ \\
\hline$<5 \mathrm{~cm}$ & 73.3 & 11 & 32.0 & 16 & \\
\hline$\geq 5 \mathrm{~cm}$ & 26.7 & 4 & 68.0 & 34 & \\
\hline Contour & & & & & $0.041^{\mathrm{a}}$ \\
\hline Round or quasi-circular & 86.7 & 13 & 58.0 & 29 & \\
\hline Lobulated & 13.3 & 2 & 42.0 & 21 & \\
\hline Margin & & & & & $0.67^{b}$ \\
\hline Well-defined & 93.3 & 14 & 84.0 & 42 & \\
\hline Ill-defined & 6.7 & 1 & 16.0 & 8 & \\
\hline Growth pattern & & & & & $0.085^{\mathrm{b}}$ \\
\hline Endoluminal & 13.3 & 2 & 36.0 & 18 & \\
\hline Exophytic or mixed & 86.7 & 13 & 64.0 & 32 & \\
\hline Calcification & & & & & $1.00^{\mathrm{b}}$ \\
\hline+ & 26.7 & 4 & 30.0 & 15 & \\
\hline- & 73.3 & 11 & 70.0 & 35 & \\
\hline Cystic change & & & & & $<0.0001^{\mathrm{a}}$ \\
\hline+ & 13.3 & 2 & 78.0 & 39 & \\
\hline- & 86.7 & 13 & 22.0 & 11 & \\
\hline Hemorrhage & & & & & $1.00^{\mathrm{b}}$ \\
\hline+ & 0 & 0 & 4.0 & 2 & \\
\hline- & 100 & 15 & 96.0 & 48 & \\
\hline Ulceration & & & & & $0.529^{\mathrm{a}}$ \\
\hline+ & 26.7 & 4 & 36.0 & 18 & \\
\hline- & 73.3 & 11 & 64.0 & 32 & \\
\hline Perilesional lymph nodes & & & & & $0.0007^{\mathrm{b}}$ \\
\hline+ & 66.7 & 10 & 18.0 & 9 & \\
\hline- & 23.3 & 5 & 82.0 & 51 & \\
\hline Tumor vessels & & & & & $0.0098^{\mathrm{a}}$ \\
\hline+ & 80.0 & 12 & 42.0 & 21 & \\
\hline- & 20.0 & 3 & 58.0 & 29 & \\
\hline Enhancement pattern & & & & & $0.0002^{\mathrm{b}}$ \\
\hline Homogeneous & 66.7 & 10 & 14.0 & 7 & \\
\hline Heterogeneous & 33.3 & 5 & 86.0 & 43 & \\
\hline Enhancement degree & & & & & $0.005^{\mathrm{a}}$ \\
\hline Light or moderate & 93.3 & 14 & 52.0 & 26 & \\
\hline Obvious & 6.7 & 1 & 48.0 & 24 & \\
\hline
\end{tabular}

${ }^{\mathrm{a}} \chi^{2}$ test; ${ }^{\mathrm{b}}$ Fisher's exact test. GISTs, gastrointestinal stromal tumors; CT, computed tomography.

Statistically significant CT features were selected for ROC analysis. The maximal area under the ROC curve is illustrated in Table III. ROC curves demonstrated in Figs. 9 and 10 indicated that lesion size, cystic change, PLNs, enhancement pattern and $\mathrm{Sa}$ value were of higher diagnostic value than others, AUC $>0.7$. Furthermore, the presence of cystic change demonstrated the best diagnostic ability, lesion size had the highest sensitivity and cystic change and Sa had the highest specificity (Table III).

\section{Discussion}

The data from the present study demonstrated that gastrointestinal schwannomas and GISTs frequently present as submucosal round or quasi-circular tumors, accompanied with hemorrhage, necrosis, cystic changes and a mild to moderate enhancement pattern. The differences between gastrointestinal schwannomas and GISTs in a number of CT features, including tumor margins and growth patterns, 
Table III. AUC of the Receiver operating characteristic curve of the computed tomography features distinguishing gastric schwannomas from gastrointestinal stromal tumors.

\begin{tabular}{lccc}
\hline Index & Specificity & Sensitivity & AUC \\
\hline Size & 0.7 & 0.9 & 0.8 \\
Contour & 0.4 & 0.9 & 0.6 \\
Cystic change & 0.9 & 0.8 & 0.8 \\
Perilesional lymph nodes & 0.7 & 0.8 & 0.7 \\
Fistula & 0.8 & 0.6 & 0.7 \\
Enhancement pattern & 0.7 & 0.9 & 0.8 \\
Ta & 0.8 & 0.7 & 0.7 \\
Sa & 0.9 & 0.6 & 0.7 \\
\hline
\end{tabular}

AUC, area under the curve; Ta, tumor arterial phase scan; Sa, tumor arterial phase scan/aorta arterial phase scan.

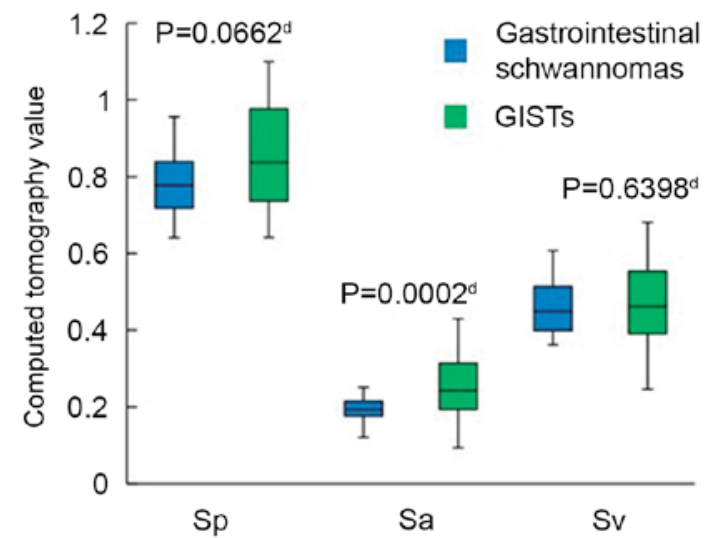

Figure 7. Comparison of standardized computed tomography values between GISTs and gastrointestinal schwannomas in plain phase, arterial phase and venous phase. Sp, Sa and Sv were all lower for gastrointestinal schwannomas when compared with GISTs and the difference in Sa was statistically significant $(\mathrm{P}=0.002)$. Sp, tumor plain phase scan/aorta plain phase scan; $\mathrm{Sa}$, tumor arterial phase scan/aorta arterial phase scan; Sv, tumor venous phase scan/aorta venous phase scan; ${ }^{\mathrm{d}}$ approximate t-test; GISTs, gastrointestinal stromal tumors.

were not statistically significant. However, the differences for other CT features, including differences in lesion size, were statistically significant, consistent with the data published by Choi et al (13). Among the statistically significant CT features, the presence of PLNs exhibited a higher predictive value in differentiating between the two types of tumor. Perilesional lymphadenopathy has been hypothesized to be induced by the inflammatory process rather than tumor metastasis $(15,16)$. Histological examination from the present study demonstrated that inflammatory cells were scattered throughout the tumors, and inflammation may stimulate the proliferation of surrounding lymph nodes. Hou et al (15) reported that the cytotoxin released by tumor cells stimulated the enlargement of lymph nodes, whereas Atmatzidis et al (16) reported that PLNs exhibited reactive inflammatory process. The data from the present study are consistent with data from Prévot et al (17), and may be explained by Atmatzidis et al (16). GISTs frequently appeared in CT images as lobulated tumors

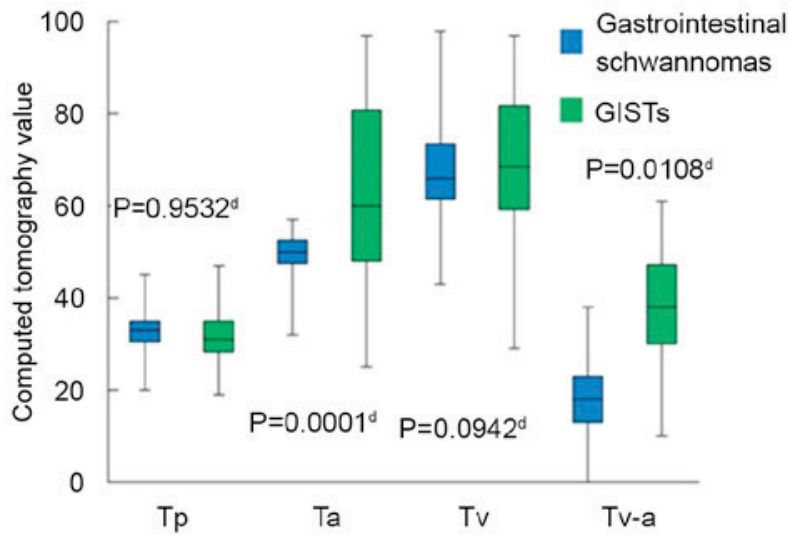

Figure 8. Comparison of tumor computed tomography values between GISTs and gastrointestinal schwannomas in plain phase, arterial phase and venous phase. Tp, Ta, Tv and Tv-a of gastrointestinal schwannomas were all lower than that of GISTs and the difference in Ta and Tv-a was statistically significant $(\mathrm{P}=0.0001 ; \mathrm{P}=0.01$, respectively). Tp, tumor plain phase; Ta, tumor arterial phase; Tv, tumor venous phase; Tv-a, tumor venous phase-tumor

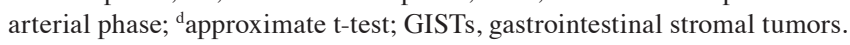

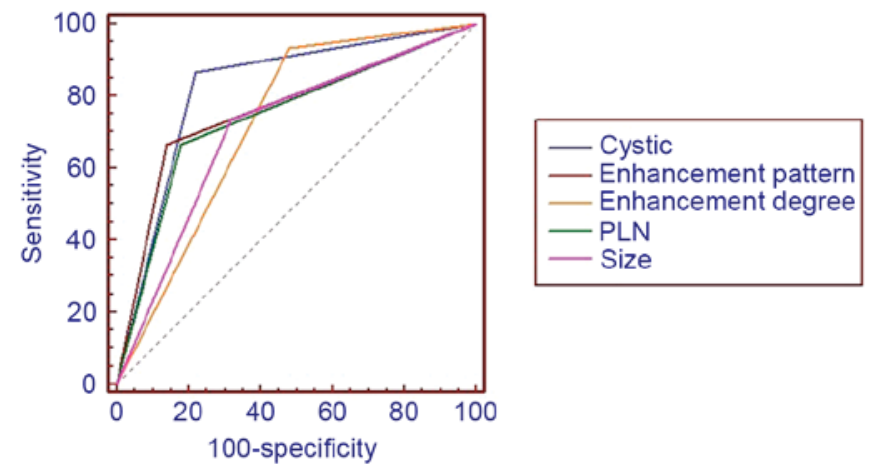

Figure 9. Receiver operating characteristic curve of qualitative data. Cystic change was the variable with the best diagnostic ability. Size demonstrated the highest sensitivity for differentiating between gastrointestinal stromal tumors and schwannomas. PLN, perilesional lymph nodes.

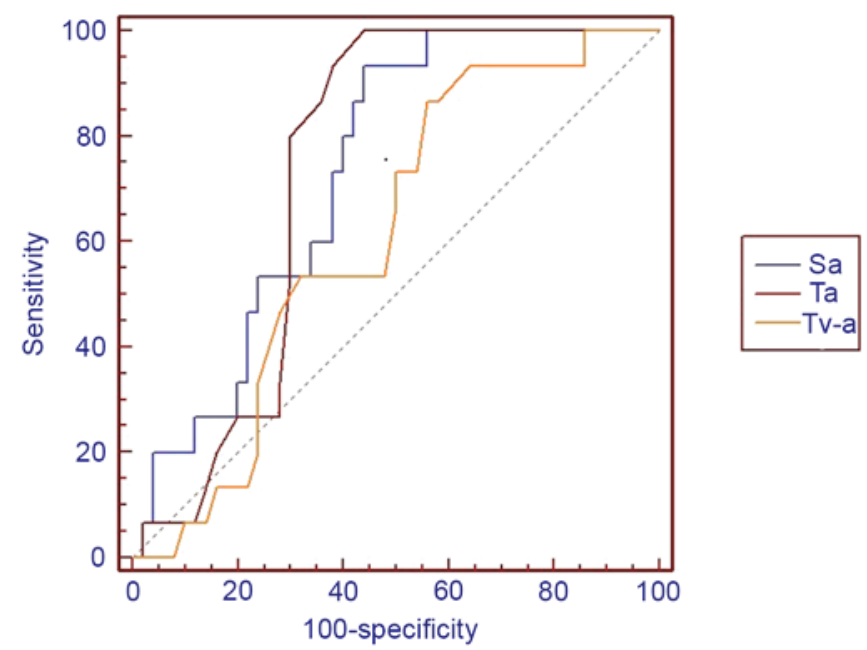

Figure 10. ROC curve of quantitative data. Sa possessed the maximal area under ROC curve and exhibited the best specificity for differentiating between gastrointestinal stromal tumors and schwannomas. ROC, receiver operating characteristic; Ta, tumor arterial phase scan; $\mathrm{Sa}, \mathrm{Ta} /$ aorta arterial phase scan; Tv-a, difference in computed tomography value between arterial and venous phases. 
with heterogeneous density and moderate to obvious heterogeneous contrast enhancement. Despite an overall abundance in blood supply in tumors compared with normal tissue, the rapid proliferation of these tumor cells led to the formation of multiple relative low-density areas with relative insufficient blood supply in an enhanced CT scan. Thus, hemorrhage, necrosis and cystic change are frequently exhibited in these tumors, as demonstrated by their heterogeneous appearances on CT (7).

In addition, the present study applied quantitative measurements to analyze the difference in absolute $\mathrm{CT}$ values and normalized CT values between gastrointestinal schwannomas and GISTs. These measurements indicated that GISTs, as invasive tumors, exhibited a more abundant blood supply when compared with benign tumors, including gastrointestinal schwannomas.

The present study also demonstrated that ROC analysis indicated that certain $\mathrm{CT}$ results, particularly cystic change, size and Sa were reliable indicators for imaging differentiation compared with the other results. Choi et al (13) and Choi et al (14) attempted to identify the CT features that may assist in distinguishing small $(<5 \mathrm{~cm})$ and large $(\geq 5 \mathrm{~cm})$ gastric GISTs from schwannomas. These studies revealed that certain CT features, including a homogenous enhancement pattern and PLNs, were more likely to indicate gastric schwannomas than GISTs. Choi et al (13) interpreted that exophytic or mixed growth and slower doubling time patterns were the most common types of CT feature associated with gastric schwannomas diagnoses. Choi et al (14) demonstrated that the pattern of enhancement, the size of the tumor, and the presence of necrosis and enlarged lymph nodes were significantly different between GISTs and schwannomas. The qualitative analysis data of the present study are consistent with these data. In addition, the present study has introduced novel quantitative parameters, which demonstrated statistically significant data, suggesting these CT features may be used to differentiate GISTs from gastrointestinal schwannomas. To the best of our knowledge, few studies have combined qualitative and quantitative analysis to distinguish between these two types of tumors.

Although certain quantitative parameters were recognized as aiding in the differentiation between the two types of tumor in the present study, specific CT features may overlap between these two tumor types in certain cases. Another limitation of the present study was the relatively small size of the group of patients with gastrointestinal schwannomas, as this type of tumor is rare. With such a limited sample size, useful cutoff CT values could not be obtained to aid CT differentiation. Thus, the clinical use of quantitative parameters to distinguish gastrointestinal Schwannomas from GIST remains impractical.

In conclusion, GISTs demonstrate the characteristics of malignant tumors, including abundant blood supply, faster growth pattern, larger volume, frequent necrosis and cystic change, which may be used to differentiate them from gastrointestinal schwannomas. Gastrointestinal schwannomas, as benign tumors, are more likely to be homogeneous with homogeneous enhancement, and cystic changes, necrosis or the presence of PLNs are relatively rare in schwannomas. Therefore, the detailed quantitative analysis of CT images, combined with qualitative analysis, will be useful in distinguishing between gastrointestinal schwannomas from GISTs.

\section{Acknowledgements}

The present study was supported by the National Natural Science Foundation of China (grant no. 81571750), the Zhujiang Scientific and Technological New Star Foundation (grant no. 2012J2200084), the Natural Science Foundation of Guangdong Province (grant nos. S2013010016004, 2014A030 311018 and 2015A030313043) and the S\&T Programs (grant no. 2014A020212125) of Guangdong Province.

\section{References}

1. Daimaru Y, Kido H, Hashimoto H and Enjoji M: Benign schwannoma of the gastrointestinal tract: A clinicopathologic and immunohistochemical study. Hum Pathol 19: 257-264, 1988.

2. Inagawa S, Hori M, Shimazaki J, Matsumoto S, Ishii H, Itabashi M, Adachi S, Kawamoto T and Fukao K: Solitary schwannoma of the colon: Report of two cases. Surg Today 31: 833-838, 2001.

3. Matsuki A, Kosugi S, Kanda T, Komukai S, Ohashi M, Umezu H, Mashima Y, Suzuki T and Hatakeyama K: Schwannoma of the esophagus: A case exhibiting high $18 \mathrm{~F}$-fluorodeoxyglucose uptake in positron emission tomography imaging. Dis Esophagus 22: E6-E10, 2009.

4. Melvin WS and Wilkinson MG: Gastric schwannoma. Clinical and pathologic considerations. Am Surg 59: 293-296, 1993

5. Miettinen M, Sarlomo-Rikala M and Lassota J: gastrointestinal stromal tumors: Recent advances in understanding of their biology. Hum Pathol 30: 1212-1220, 1999

6. Hong HS, Ha HK, Won HJ, Byun JH, Shin YM, Kim AY, Kim PN, Lee MG, Lee GH and Kim MJ: Gastric schwannomas: Radiological features with endoscopic and pathological correlation. Clin Radiol 63: 536-542, 2008.

7. Levy AD, Remotti HE, Thompson WM, Sobin LH and Miettinen M: Gastrointestinal stromal tumors: Radiologic features with pathologic correlation. Radiographics 23: 283-304, 456; quiz 532, 2003 .

8. Loffeld RJ, Balk TG, Oomen JL and van der Putten AB: Upper gastrointestinal bleeding due to a malignant Schwannoma of the stomach. Eur J Gastroenterol Hepatol 10: 159-162, 1998.

9. Watanabe A, Ojima H, Suzuki S, Mochida Y, Hirayama I, Hosouchi Y, Nishida Y, Kashiwabara K, Ohno T, Mochiki E and Kuwano H: An individual with gastric schwannoma with pathologically malignant potential surviving two years after laparoscopy-assisted partial gastrectomy. Case Rep Gastroenterol 5: 502-507, 2011.

10. Zuo HD, Zhang XM and Zhai ZH: Nerve Sheath tumor of stomach: Two Cases Report. J Med Cases 3: 68-72, 2012.

11. Ludwig DJ and Traverso LW: Gut stromal tumors and their clinical behavior. Am J Surg 173: 390-394, 1997.

12. Janowitz P, Meier F and Reisig J: Gastric schwannoma as a rare differential diagnosis of pleural effusion. Z Gastroenterol 40: 925-928, 2002.

13. Choi JW, Choi D, Kim KM, Sohn TS, Lee JH, Kim HJ and Lee SJ: Small submucosal tumors of the stomach: Differentiation of gastric schwannoma from gastrointestinal stromal tumor with CT. Korean J Radiol 13: 425-433, 2012.

14. Choi YR, Kim SH, Kim SA, Shin CI, Kim HJ, Kim SH, Han JK and Choi BI: Differentiation of large $(\geq 5 \mathrm{~cm})$ gastrointestinal stromal tumors from benign subepithelial tumors in the stomach: Radiologists' performance using CT. Eur J Radiol 83: 250-260, 2014.

15. Hou YY, Tan YS, Xu JF, Wang XN, Lu SH, Ji Y, Wang J and Zhu XZ: Schwannoma of the gastrointestinal tract: A clinicopathological, immunohistochemical and ultrastructural study of 33 cases. Histopathology 48: 536-545, 2006.

16. Atmatzidis S, Chatzimavroudis G, Dragoumis D, Tsiaousis P, Patsas A and Atmatzidis K: Gastric schwannoma: A case report and literature review. Hippokratia 16: 280-282, 2012.

17. Prévot S, Bienvenu L, Vaillant JC and de Saint-Maur PP: Benign schwannoma of the digestive tract: A clinicopathologic and immunohistochemical study of five cases, including a case of esophageal tumor. Am J Surg Pathol 23: 431-436, 1999. 